\title{
Nursing Activities Score (NAS) como instrumento para medir carga de trabalho de enfermagem em UTI adulto*
}

\author{
EVALUATION OF THE NURSING ACTIVITIES SCORE (NAS) AS A NURSING WORKLOAD \\ MEASUREMENT TOOL IN AN ADULT ICU
}

\section{NURSING ACTIVITIES SCORE - NAS COMO INSTRUMENTO PARA MEDIR LA CARGA DE TRABAJO DE ENFERMERÍAEN UCIADULTO}

\author{
Regina Maria Yatsue Conishi ${ }^{1}$, Raquel Rapone Gaidzinski²
}

\section{* Extraído da disser- tação "Avaliação do NAS - Nursing \\ Activities Score - como instrumento de medida de carga de trabalho de enferma- gem em UTI geral adulto", Escola de Enfermagem, \\ Universidade de São Paulo(EEUSP), 2005. \\ 1 Enfermeira Coorde- \\ nadora de Enfermagem \\ - UTI do Hospital \\ Sírio Libanês. Mestre em enfermagem pela EEUSP - São Paulo, SP, Brasil. \\ rconishi@yahoo.com.br \\ 2 Enfermeira. Professora \\ Associada do Depar- \\ tamento de Orientação \\ Profissional da \\ EEUSP- São Paulo, \\ SP, Brasil. \\ raqui@usp.br}

\section{RESUMO}

Pesquisa de campo, prospectiva, quantitativa, descritiva-exploratória, realizada na UTI geral/ adulto de um hospital privado do município de São Paulo. Objetivos: avaliar o NAS - Nursing Activities Score - como medida de carga de trabalho de enfermagem; sua aplicabilidade por turnos e sua correspondência com o quantitativo de enfermagem efetivo. Classificados 33 pacientes: idade média: 70,4 anos (+/-16,5), 66,7\% do sexo masculino; permanência média na UTI: 17 dias (+/- 20,4); SAPSII: 41,7 $(+/-17,9)$; risco de morte: (RM) $33,5 \%$ (+/- 26,8); 63,6\% transferidos para Unidades de Cuidados Semi-Intensivos, 18,2\% evoluíram a óbito. Obtiveram-se 396 medidas por turnos (134manhã; 132-tarde; 130-noturno), média de 55,4 (+/-12,3) e 147 medidas de NAS de 24h, média de 69,6 (+/-18,2). O instrumento mostrou-se mais adequado à aplicação em 24 horas que por turnos, tendendo a refletir o número de profissionais efetivo, revelando-se interessante instrumento de classificação de pacientes e carga de trabalho de enfermagem em terapia intensiva.

\section{DESCRITORES}

Unidades de Terapia Intensiva. Pacientes/classificação.

Recursos humanos de enfermagem no hospital. Carga de trabalho.

\section{ABSTRACT}

This is an exploratory, descriptive, prospective field study with a quantitative approach carried out at a general/adult Intensive Care Unit in the city of São Paulo. Objectives: to evaluate the NAS Nursing Activities Score - as a tool for measuring nursing workload, its use in measuring shifts, and its correspondence to the number of effective nursing personnel. Thirty-three patients, with mean age of 70 years (+/-16.5), were classified. Most were males (66.7\%). Length of stay in the ICU was 17 days (+/- 20.4); SAPSII was $41.7(+/-17.9)$, with mean probability of death of $33.5 \%(+/$ - 26.8); 63.6\% were transferred to Intermediate Care Units and 18.2\% died during ICU stay. Three hundred and ninety six NAS measures (shifts) were performed (134-morning, 132-afternoon, 130-evening), with averages of $55.4(+/-12.3)$ and 147 in 24-hour NAS and mean of 69.6 (+/- 18.2). NAS performed better in 24-hour application than in shifts, and proved to be an interesting tool for patient and nursing workload classification in intensive care.

\section{KEY WORDS}

Intensive Care Units.

Patients/classification.

Nursing staff, hospital.

Workload.

\section{RESUMEN}

Pesquisa de campo, prospectiva, cuantitativa, descriptivo-exploratoria desarrollado en una UCI (Unidad de Cuidados Intensivos) general/adultos en el municipio de São Paulo. Objetivos: evaluar el NAS como instrumento para medir carga de trabajo de enfermeras, su aplicación en períodos y su correspondencia con el número efectivo de enfermeros. 33 pacientes con edad media de 70,4 años (+/-16,5) fueron clasificados; $66,7 \%$ del sexo masculino; media de permanencia en UCI, 17 días (+/-20,4); SAPSII 41,7 (+/-17,9); riesgo de muerte (RM), 33,5\% $(+/-26,8) .63,6 \%$ recibieron alta para unidades de cuidados semi intensivos y 18,7\% murieron. Se lograron 396 medidas de NAS en períodos (134-mañana; 132tarde; 130-noche), media: 55,4 (+/ -12,3); y 147 medidas de 24 horas, media: 69,6 puntos (+/$18,2)$. NAS se mostró más adecuado a aplicación en 24 horas, tendiendo a reflexionar en el número de enfermeros en efectivo y se reveló interesante instrumento de clasificación de pacientes y en la carga de trabajo de enfermeras en UCI.

\section{DESCRIPTORES}

Unidades de Terapia Intensiva. Pacientes/clasificación.

Personal de enfermería en hospital. Carga de trabajo. 


\section{INTRODUÇÃO}

A evolução tecnológica nas Unidades de Terapia Intensiva (UTI) tem influenciado na mudança do perfil dos pacientes internados nas terapias intensivas com conseqüências no tempo de permanência e nível de atenção requerido, pois com mais recursos terapêuticos e tecnológicos à disposição, os pacientes tornaram-se mais graves e mais complexos para serem cuidados, necessitando de profissionais mais qualificados ${ }^{(1-3)}$

Outro fator que ganha força nesses últimos anos é a humanização da assistência hospitalar, sobretudo nas UTIs, exigindo que os profissionais envolvidos dediquem maior tempo na atenção às necessidades de apoio emocional e informação ao paciente e familiares. Essa condição passa a conferir valor agregado ao produto - serviço de saúde fazendo parte da missão e dos projetos de marketing das instituições ${ }^{(4)}$.

Conseqüentemente, essas demandas requerem mudanças na qualificação e quantificação dos profissionais que atuam na área. Paralelamente, a crise econômica que recai sobre todos os setores da sociedade atinge, também, a prestação de serviços de saúde. Nos últimos anos, grande ênfase se tem dado ao aumento da demanda e dos custos em saúde e, por conseguinte, grandes esforços têm sido despendidos no sentido de se reduzir custos através do uso racional dos recursos existentes.

Nesse contexto, as UTIs tornaram-se uni-dades onde se concentram recursos humanos e tecnológicos altamente especializados que proporcionam assistência considerada como das mais complexas, sofisticadas e onerosas do sistema de atendimento à saúde ${ }^{(5-6)}$.

Em meio a um cenário empresarial mais competitivo( ${ }^{(7)}$, em busca de recursos escassos, os gestores das organizações de saúde precisam utilizar instrumentos gerenciais para administrar os recursos utilizados na consecução das atividades operacionais sem os quais tornar-se-iam inviáveis. Assim, a crescente preocupação com custos e a própria necessidade de fundamentação para negociação, vem levando os profissionais que gerenciam serviços de saúde a se envolverem de forma mais direta com o conhecimento da performance financeira de seus serviços, pois segundo um estudo $^{(7)}$,

o melhor gestor de custos é quem conhece profundamente as operações e não quem reúne apenas os conceitos relacionados à contabilização dos custos.

A equipe de enfermagem representa, na maioria das instituições de saúde, o percentual quantitativo e orçamentário mais significativo, tornando-se a equipe mais visada quando o problema é redução de $\operatorname{custos}^{\left({ }^{()}\right.}$. Nessa direção a enfermagem vem argumentando para provar, atualmente de forma mais fundamentada, que a inadequação numérica e qualitativa dos recursos de enfermagem lesa a clientela no seu direito de assistência à saúde livre de riscos e pode comprometer legalmente a instituição pelas falhas ocorridas devido à sobrecarga de trabalho e à deficiência da qualidade da assistência prestada ${ }^{(9)}$.

Com a finalidade de auxiliar na avaliação da adequação qualitativa e quantitativa dos recursos humanos de enfermagem com respeito à carga de trabalho, têm-se realizado vários estudos para classificar os pacientes em relação às suas necessidades de enfermagem bem como quantificar o tempo de assistência de enfermagem despendido.

O presente estudo versa sobre a utilização do Nursing Activities Score (NAS), instrumento desenvolvido por Miranda e seus colaboradores ${ }^{(10)}$, que visa medir o tempo de assistência de enfermagem em UTI.

Os autores ${ }^{(10)}$ desenvolveram o NAS a partir do Therapeutic Intervention Scoring System (TISS-28) ${ }^{(11)}$ para tornálo mais representativo das atividades realizadas pela enfermagem na UTI. A mudança mais substancial ocorreu na categoria das atividades básicas, que foi sub-categorizada em: monitorização e controles, procedimentos de higiene; mobilização e posicionamento; suporte e cuidados aos familiares e pacientes e tarefas administrativas e gerenciais. $\mathrm{O}$ instrumento resultante consta de 7 grandes categorias e 23 itens. Cada item possui uma pontuação, portanto o escore atribuído a um paciente resulta da soma das pontuações dos itens que correspondem às necessidades de assistência direta e indireta dos pacientes. Esse escore representa quanto tempo de um profissional de enfermagem o paciente requereu nas últimas 24 horas. Assim se a pontuação for 100 , interpreta-se que o paciente requereu $100 \%$ do tempo de um profissional de enfermagem no seu cuidado nas últimas 24 horas. O NAS foi traduzido para o português e validado por Queijo(5).

\section{OBJETIVOS}

\section{Geral:}

- avaliar a aplicabilidade do NAS como instrumento de medida de carga de trabalho da equipe de enfermagem em UTI geral-adulto.

\section{Específicos:}

- caracterizar a população do estudo demograficamente e segundo a gravidade; 
- classificar os pacientes, segundo o NAS e analisar a relação do escore NAS com o quantitativo de profissionais de enfermagem que estavam prestando assistência na UTI.

\section{MÉTODO}

Trata-se de uma pesquisa de campo, prospectiva, de abordagem quantitativa do tipo descritivo-exploratória, realizada em UTI geral-adulto, com dez leitos de um hospital geral com 240 leitos da rede privada do município de São Paulo.

A UTI, campo de estudo, conta com atendimento contínuo de enfermagem, fisioterápico e médico. Trabalham por turno, duas a três enfermeiras. A equipe de enfermagem conta somente com técnicos de enfermagem, como profissionais de nível médio, sendo cinco técnicos de enfermagem por turno, cada técnico de enfermagem assiste, no máximo, a dois pacientes. A assistência de enfermagem é sistematizada, sob referencial teórico de Wanda Horta, com três fases do processo de enfermagem implantadas: histórico, prescrição e evolução de enfermagem.

A equipe da UTI conta, também, com agentes de transporte e cuidados com materiais $24 \mathrm{~h}$ por dia, capacitados para cuidar dos materiais e equipamentos da unidade e apoiar os demais profissionais nos transportes dos pacientes da UTI. Auxiliares administrativos realizam, primordialmente, pedidos de farmácia, almoxarifado e laboratório e lançamentos de débitos nas contas dos pacientes.

Os dados da pesquisa foram coletados após aprovação pela Comissão de Ética em Pesquisa da Instituição.

O estudo ocorreu em dois períodos de sete dias (de 24 a 30 de junho de 2004 e de 9 a 16 de setembro de 2004). Foram incluídos no estudo todos os pacientes adultos que estavam internados na UTI no primeiro dia de cada período do estudo e todos os pacientes que internaram, seqüencialmente, até o sétimo dia, independentemente do diagnóstico e do tempo de permanência e que pessoalmente ou por intermédio de seus representantes, concordaram com os termos desta pesquisa, mediante assinatura do Termo de Consentimento Livre e Esclarecido.

Com a finalidade de caracterizar a população do estudo, foram coletados no prontuário de cada paciente os dados demográficos, de internação, gravidade (SAPSII - Simplified
Acute Physiology Score) ${ }^{(12)}$ e risco de morte (RM). Os dados do SAPSII foram lançados em uma planilha eletrônica disponibilizada via Internet ${ }^{(13)}$, que efetuou o cálculo do índice e risco de morte.

Durante os 14 dias do estudo, os pacientes que estiveram na unidade foram classificados quanto ao NAS, ao final de cada turno, segundo o registro, no prontuário do paciente, das atividades desenvolvidas pelos profissionais de enfermagem. A partir da classificação por turnos, obteve-se a classificação de 24h para os pacientes que completavam 24h na unidade. Os dados foram lançados em planilhas eletrônicas para cálculo dos índices.

As informações do NAS que normalmente não se encontram registradas, como aquelas relacionadas ao tipo e duração de atendimento aos familiares, atividades administrativas e número de pessoas envolvidas nas atividades foram obtidas ao final de cada turno, dos próprios profissionais de enfermagem que cuidaram de cada paciente.

\section{RESULTADOS E DISCUSSÃO}

Os resultados estão apresentados, atendendo aos objetivos propostos, conforme a seqüência:

- Caracterização dos pacientes incluídos no estudo, segundo dados demográficos e gravidade;

- Classificação dos pacientes, segundo a carga de trabalho de enfermagem obtida pelo NAS por turno e em 24h;

- Análise da relação da pontuação NAS com o quantitativo de profissionais de enfermagem presentes na UTI.

\section{Caracterização dos pacientes}

Um total de 35 pacientes esteve presente na unidade nos dois períodos do estudo, dos quais, dois foram excluídos: o primeiro por ser pediátrico (permaneceu na unidade por 11 horas até ser transferido), e o segundo, por não ter sido possível obter o termo de consentimento. Assim, a amostra final do estudo foi composta por 33 pacientes.

Observa-se que nos períodos do presente estudo a ocupação esteve elevada, acima de 90\% (Figura 1) em média, oferecendo a possibilidade de se avaliar a dinâmica de trabalho da unidade muito próximo à sua capacidade máxima. 


\section{ocupação (\%)}

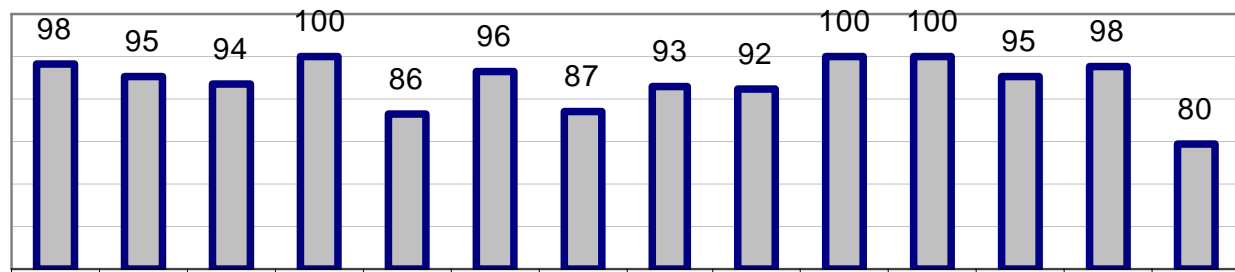

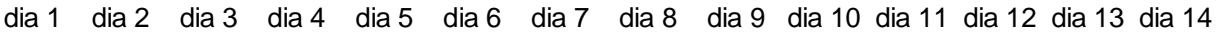

dia do estudo

Figura 1 - Taxa de ocupação média diária da UTI nos períodos de 24 a 30 de junho e 9 a 16 de setembro - São Paulo - 2004

Em 3 dias, a taxa de ocupação foi de 100\%; 8 dias tiveram taxa de ocupação entre 90 e $99 \%$, e outros 3 dias tiveram taxa de ocupação abaixo de $90 \%$.

A média de idade dos pacientes foi 70,4 anos (+/- 16,5 anos), variando de 33 a 92 anos, podendo ser considerada uma população idosa com predomínio de pacientes do sexo masculino, 66,7\% ( $\mathrm{n}=22)$. O tempo médio de permanência na UTI foi de 17 dias (+/-20,4 dias), variando de 0,4 a 86,1 dias. Considerando que a instituição em estudo dispõe de unidades de cuidados semi-intensivos, a alta média de permanência pode ter sido devida, à gravidade dos pacientes que compuseram a amostra.

O SAPSII médio da população foi de 41,7 (+/- 17,9, IC95\% = 6) com pontuação mínima de 6 e máxima de 84 . O risco de morte (RM) médio foi de 33,5\% (+/- 26,8; IC95\%=9) sendo mínimo de $0,5 \%$ e o máximo de $94,6 \%$, podendo ter sido influenciado pela característica etária da população.

O tipo de internação predominante foi clínico, 60,6\% $(n=20)$ sendo o cirúrgico 39,4 ( $n=13)$ e, destes, 21,2\% (n=7) pós-operatórios de cirurgias eletivas e 18,2\% (n=6) pós-operatórios de cirurgia de emergência.

Quanto à procedência, 27,3\% dos pacientes (n=9) vieram do Centro Cirúrgico (CC). Outros 27,3\% (n=9) foram admitidos vindos do Pronto Atendimento (PA). Foram transferidos das Unidades Semi-Intensivas (UCSI), 8 pacientes (24,2\%). Das Unidades de Internação (UI), foram transferidos 6 pacientes (18,2\%) e somente 1 paciente, 3,0\%, veio transferido de outro hospital. Ocorreu equilíbrio entre as procedências de CC e PA, maioria na amostra. UCSI e UI, também tiveram participações semelhantes como origem de pacientes para a UTI.
Quanto ao destino, 63,6\% ( $\mathrm{n}=21)$ foram transferidos para as UCSI após a alta da UTI; $15,2 \%(n=5)$ para UI. Um paciente $(3 \%)$, recebeu alta para casa. Seis pacientes evoluíram a óbito, $18,2 \%$, taxa menor que a prevista pelo RM-SAPSII médio, 33,5\%.

\section{Classificação dos pacientes, segundo a carga de trabalho de enfermagem obtida pelo NAS por turno e em $24 \mathrm{~h}$}

Na amostra de 33 pacientes deste estudo, o NAS foi aplicado 147 vezes, considerando as medidas para 24h, tendo sido incluídos nas aplicações do instrumento NAS, as pontuações de dias incompletos, nos quais o paciente deixou a unidade ou foi admitido em qualquer momento, entre 7h00min de um dia e 6h59min do dia seguinte. Optou-se pela inclusão destas medidas, pois, por se tratar de um instrumento para quantificar tempo de assistência de enfermagem, ocorreria uma sub-quantificação de trabalho, caso fossem excluídos, já que o atendimento é realizado até a saída física do paciente da unidade ou a partir de sua admissão.

Conforme expresso na Tabela 1 , a pontuação NAS que considerou pacientes com permanência de 24 horas completas + incompletas variou de 22,3 a 127,9 com média de 65,5 (+/- 18,8). As medidas do NAS de 24 horas dos pacientes com permanência de 24 horas incompletas apresentaram menor valor médio, 49,5 pontos $(+/-10,5$; min.= 22,3; máx.= 79,5 pontos), em relação aos NAS de 24 horas dos pacientes com permanência de 24h completas, que tiveram média de 69,6 (+/- 18,2; min.= 27,9; máx.=127,9 pontos). Queijo ${ }^{(5)}$ obteve pontuação NAS média de 67,1 (+/- 8,42; min.= 55,7; max.=107,2), para pacientes com permanência de $24 \mathrm{~h}$ completas. 
Tabela 1 - Pontuações dos NAS de 24h para pacientes internados nos períodos de 24 a 30 de junho e 9 a 16 de setembro, segundo a permanência - São Paulo - 2004

\begin{tabular}{crcccccr}
\hline & & & & \multicolumn{3}{c}{ Pontuação NAS 24h } \\
Permanência 24h & N & $\%$ & média & DP & IC & Mínimo & Máximo \\
\hline Completa & 117 & 80 & 69,6 & 18,2 & 3,3 & 27,9 & 127,9 \\
Incompleta & 30 & 20 & 49,5 & 10,5 & 3,7 & 22,3 & 79,5 \\
Completa + incompleta & 147 & 100 & 65,5 & 18,8 & 3,0 & 22,3 & 127,9 \\
\hline
\end{tabular}

Embora menor, o NAS médio, de pacientes com permanência de 24h incompletas indica tempo de assistência considerável enquanto o paciente permanece na unidade, em média 50\% do tempo de um profissional de enfermagem. Estes pacientes normalmente são excluídos de indicadores de produção, como taxa de ocupação e outros instrumentos que desconsideram informações sobre permanências menores que 24h. No entanto, cabe ressaltar que embora as internações com tempo menor que $24 \mathrm{~h}$ possam interferir na carga de trabalho dos turnos na unidade, para efeitos de dimensionamento de profissionais devem ser desconsi-deradas, pois como o valor da pontuação NAS será menor, esses dados, se considerados irão baixar o valor médio NAS da amostra, o que pode ser evidenciado nos dados da Tabela 1 .

O Quadro 1 apresenta o perfil de pontuação do NAS de 24h dos pacientes, com freqüência de pontuação em cada item e sub-item.

Verificou-se, neste estudo, que os itens/sub-itens mais freqüentemente pontuados individualmente (acima de 80\%) foram: 3 - Medicação (99,3\%), 17- Medida quantitativa do débito urinário (95,9\%), 2- Investigações laboratoriais (91,2\%), 8a- Realização de processamento de dados clínicos, solicitação de exames, troca de informações profissionais (89,1\%), 7a- Suporte e cuidado aos familiares e pacientes que requerem dedicação exclusiva por cerca de uma hora em algum plantão (87,8\%), 1a - Sinais vitais horários, cálculos e registro regular do balanço (85,7\%), 9 - Suporte respiratório (81,6\%).

Nenhum item/sub-item foi pontuado em 100\% das medidas. Nota-se que no grupo das atividades básicas, as freqüências de pontuação dos sub-itens dos itens 4 e 8 somam $100 \%$, o que indica terem sido atividades executadas todos os dias em todos os pacientes, diferindo apenas em intensidade. Já os cuidados/atividades que constam nos sub-itens dos itens 1 e 6, assim como, o item 3, que espera-se que todos pacientes recebam, não somam 100\%, pois em cada um deles, um paciente não obteve pontuação, todos em medidas de 24 horas incompletas.

Quanto aos itens menos pontuados, encontram-se, com freqüência abaixo de $10 \%$ os itens/sub-itens 14 - Monitorização do átrio esquerdo (0,7\%); 19 - Tratamento da acidose/ alcalose metabólica complicada (0,7\%); 1c - Presença à beira do leito e observação ou atividade contínua por 4 horas ou mais em algum plantão (1,4\%); 13 - Reposição intravenosa de grandes perdas de fluídos (1,4\%); 8c - Realização de tarefas administrativas e gerenciais que requerem dedicação integral por cerca de 4 horas ou mais de tempo em algum plantão (2,0\%); 22 - Intervenções específicas na unidade de terapia intensiva (7,5\%); 6 a - Realização de mobilização e posicionamento até 3 vezes em 24 horas (8,8\%); 8b - Realização de tarefas administrativas e gerenciais que requerem dedicação integral por cerca de 2 horas em algum plantão (8,8\%); $7 \mathrm{~b}$ - Suporte e cuidado aos familiares e pacientes que requerem dedicação exclusiva por 3 horas ou mais em algum plantão (9,5\%); 20 - Hiper-alimentação intravenosa (9,5\%).

Os itens 15 - Reanimação cardio-respiratória e 18 - Medida de pressão intracraniana, não foram pontuados neste estudo. Vale ressaltar que dos 6 óbitos que constam na caracterização da amostra, 2 ocorreram durante o período de coleta do NAS e, nestes, não foi realizada reanimação.

$\mathrm{O}$ item 1c indica que o paciente teve presença contínua por 4 horas ou mais da enfermagem para observação e/ou controle nas $24 \mathrm{~h}$ estabelecidas para medida. Isso, na prática diária, muitas vezes observa-se como necessidade nem sempre atendida devido à demanda da unidade e o quadro de pessoal disponível. No presente estudo foi notada, neste item, tendência clara à pontuação espontânea do sub-item 1c, pelos profissionais de enfermagem que prestavam assistência, mas, ao se questionar o caráter da continuidade e do efetivamente realizado, revertiam-se, principalmente, para o sub-item 1a. Isso talvez tenha sido reflexo de uma sensação de continuidade ou mesmo de uma demanda sentida pelo profissional e talvez aponte para uma necessidade de maior elucidação deste item.

O item 4 refere-se à realização de procedimentos de higiene, incluindo troca de curativos e troca de roupa de cama e seus sub-itens foram definidos em relação à duração dessas atividades somadas ao longo do dia. Na unidade em que se realizou o presente estudo, a enfermagem realiza a troca de todos os curativos, mesmo cirúrgicos, e a troca de roupa de cama costuma ocorrer pelo menos duas vezes ao dia para promoção de conforto e manutenção da área de apoio dorsal livre de umidade. O banho é realizado, em geral uma vez ao dia, à noite e a higiene íntima sempre que necessário. Ainda, pacientes que recebiam dieta enteral apresentavam maior freqüência de evacuações diárias, acarretando procedimentos de higiene em maior número. 
Quadro 1 - Freqüência de pontuação de cada item e sub-item no NAS de 24h para pacientes internados na UTI nos períodos de 24 a 30 de junho e 9 a 16 de setembro - São Paulo - 2004

\begin{tabular}{|c|c|c|}
\hline ITENS E SUB-ITENS (RESUMIDOS) & $\mathbf{n}$ & $\%$ \\
\hline 1a. Sinais vitais horários, cálculos e registro regular do balanço hídrico. (4,5 pts) & 126 & 85,7 \\
\hline 1b. Presença à beira do leito e observação ou atividade contínua por $2 \mathrm{~h}$ ou mais. (12,1 pts) & 18 & 12,2 \\
\hline 1c. Presença à beira do leito e observação ou atividade contínua por 4 h ou mais. $(19,6$ pts $)$ & 2 & 1,4 \\
\hline 2. Investigações laboratoriais: bioquímica e microbiológicas (4,3pts) & 134 & 91,2 \\
\hline 3. Medicação, exceto drogas vasoativas (5,6 pts) & 146 & 99,3 \\
\hline 4a. Realização de procedimentos de higiene. (4,1 pts) & 92 & 62,6 \\
\hline 4b. Realização de procedimento de higiene que durem mais do que 2 h. (16,5 pts) & 40 & 27,2 \\
\hline 4c. Realização de procedimentos de higiene que durem mais do que 4 h. (20 pts) & 15 & 10,2 \\
\hline 5. Cuidados com drenos - Todos (exceto sonda gástrica) (1,8 pts) & 30 & 20,4 \\
\hline 6a. Realização do(s) procedimento(s) de mobilização e posicionamento até 3 vezes em 24 h. (5,5 pts) & 13 & 8,8 \\
\hline $\begin{array}{l}\text { 6b. Realização do(s) procedimento(s) mais do que } 3 \text { vezes em } 24 \text { h ou com } 2 \text { enfermeiros em qualquer frequência. } \\
(12,4 \text { pts) }\end{array}$ & 73 & 49,7 \\
\hline 6c. Realização do(s) procedimento(s) com 3 ou mais enfermeiros em qualquer freqüência. (17,0 pts) & 60 & 40,8 \\
\hline $\begin{array}{l}\text { 7a. Suporte e cuidado aos familiares e pacientes que requerem dedicação exclusiva por cerca de uma hora em algum } \\
\text { plantão. ( } 4,0 \text { pts) }\end{array}$ & 129 & 87,8 \\
\hline $\begin{array}{l}\text { 7b. Suporte e cuidado aos familiares e pacientes que requerem dedicação exclusiva por } 3 \mathrm{~h} \text { ou mais em algum } \\
\text { plantão. (32,0 pts) }\end{array}$ & 14 & 9,5 \\
\hline 8a. Realização de tarefas administrativas e gerenciais de rotina. ( 4,2 pts) & 131 & 89,1 \\
\hline $\begin{array}{l}\text { 8b. Realização de tarefas administrativas e gerenciais que requerem dedicação integral por cerca de } 2 \mathrm{~h} \text { em algum } \\
\text { plantão. }(23,2 \text { pts) }\end{array}$ & 13 & 8,8 \\
\hline $\begin{array}{l}\text { 8c. Realização de tarefas administrativas/gerenciais que requerem dedicação integral por cerca de } 4 \mathrm{~h} \text { ou mais de } \\
\text { tempo em algum plantão. (30,0 pts) }\end{array}$ & 3 & 2,0 \\
\hline $\begin{array}{l}\text { 9. Suporte respiratório. Qualquer forma de ventilação mecânica/ventilação assistida; oxigênio suplementar por } \\
\text { qualquer método (1,4 pts) }\end{array}$ & 120 & 81,6 \\
\hline 10. Cuidado com vias aéreas artificiais. Tubo endotraqueal ou cânula de traqueostomia (1,8 pts) & 76 & 51,7 \\
\hline $\begin{array}{l}\text { 11. Tratamento para melhora da função pulmonar. Fisioterapia torácica, espirometria estimulada, terapia inalatória, } \\
\text { aspiração endotraqueal. }(4,4 \text { pts) }\end{array}$ & 94 & 63,9 \\
\hline 12. Medicação vasoativa independente do tipo e dose. (1,2 pts) & 47 & 32,0 \\
\hline $\begin{array}{l}\text { 13. Reposição intravenosa de grandes perdas de fluídos. Administração de fluídos }>31 / \mathrm{m} 2 / \text { dia independente do tipo } \\
\text { de fluido administrado( } 2,5 \text { pts) }\end{array}$ & 2 & 1,4 \\
\hline 14. Monitorização do átrio esquerdo, com ou sem medida de débito cardíaco.(1,7 pts) & 1 & 0,7 \\
\hline 15. Reanimação cardiorrespiratória nas últimas 24 h (excluído soco precordial) (7,1pts) & 0 & 0,0 \\
\hline 16. Técnicas de hemofiltração. Técnicas dialíticas (7,7 pts) & 21 & 14,3 \\
\hline 17. Medida quantitativa do débito urinário (ex sonda vesical de demora) (7,0 pts) & 141 & 95,9 \\
\hline 18. Medida de pressão intracraniana (1,6 pts) & 0 & 0,0 \\
\hline 19. Tratamento da acidose/alcalose metabólica complicada (1,3 pts) & 1 & 0,7 \\
\hline 20. Hiperalimentação intravenosa (2,8 pts) & 14 & 9,5 \\
\hline 21. Alimentação enteral. Através de tubo gástrico ou outra via gastrintestinal (ex: jejunostomia) (1,3 pts) & 73 & 49,7 \\
\hline 22. Intervenções específicas na unidade de terapia intensiva. (2,8 pts) & 11 & 7,5 \\
\hline 23. Intervenções específicas fora da unidade de terapia intensiva. Procedimentos diagnósticos ou ci & 14 & 9,5 \\
\hline
\end{tabular}

O sub-item $6 \mathrm{~b}$ e $6 \mathrm{c}$ obtiveram maiores freqüências de pontuação, $49,7 \%$ e $40,8 \%$ respectivamente, sendo que o sub-item 6 a foi o menos pontuado neste item, $8,8 \%$ das medidas. Este item refere-se às atividades de mobilização e posicionamento e, no serviço campo do estudo, por questões ergonômicas e de segurança para o paciente, as mobi- lizações e posicionamento são realizadas no mínimo por duas pessoas e os decúbitos são prescritos para serem realizados a cada 2 horas. No item 6a foram pontuados somente os pacientes que se movimentavam espontaneamente, necessitando somente do auxílio de apenas um profissional. 
O item 21 - administração de dieta enteral - foi pontuado em $49,7 \%$ das medidas. Este item é altamente dependente e relacionado com as diretrizes terapêuticas adotadas pelas equipes médicas, dos processos mórbidos e de outras características apresentadas pelo grupo de pacientes estudados, como idade, tipo de internação e tempo de permanência na UTI.

\section{Relação da pontuação NAS com o número de profissionais de enfermagem presentes na UTI}

O período de 14 dias do estudo resultou em 42 turnos: 14 turnos da manhã (M), das 7às 13h, 14 turnos da tarde (T) das 13 às 19 h e 14 turnos noturnos $(\mathrm{N})$, das 19 às 7h. Quanto ao número de profissionais de enfermagem, verifica-se que em 4 turnos $(9,5 \%)$ trabalharam 6 pessoas, sempre 4 técnicos e 2 enfermeiras. Em 10 turnos (23,8\%) houve 8 profissionais de enfermagem trabalhando, sempre 3 enfermeiras e 5 técnicos de enfermagem. Na maior parte dos turnos, $66,7 \%$, $(n=28)$, havia 7 profissionais de enfermagem trabalhando, 2 enfermeiras e 5 técnicos de enfermagem ou 3 enfermeiras e 4 técnicos de enfermagem ou seja $29 \%$ de enfermeiras e $71 \%$ de técnicos ou $43 \%$ de enfermeiras e 57\% técnicos.

O quadro efetivo diário foi, em média de 21,4 profissionais de enfermagem, sendo que, no plantão da manhã trabalharam em média 7,5 profissionais, à tarde, 7,1, à noite 6,9. A média geral foi de 7,1 profissionais por turno.

Quanto à relação paciente/profissional de enfermagem verifica-se conforme Tabela 2 a relação variou de 1 a 1,5 pacientes por profissional de enfermagem nos turnos, tendo sido predominante a relação 1,4 pacientes por profissional de enfermagem (54,8\% dos turnos).

Tabela 2 - Distribuição dos turnos conforme o número de pacientes por profissional de enfermagem efetivamente trabalhando nos períodos de 24 a 30 de junho e 9 a 16 de setembro - São Paulo - 2004

\begin{tabular}{ccc}
\hline $\begin{array}{c}\text { Relação paciente/profissional } \\
\text { de Enfermagem por turno }\end{array}$ & $\mathrm{N}$ & $\%$ \\
\hline 1 & 1 & 2,4 \\
1,1 & 4 & 9,5 \\
1,2 & 3 & 7,1 \\
1,3 & 10 & 23,8 \\
1,4 & 23 & 54,8 \\
1,5 & 1 & 2,4 \\
\hline Total & 42 & 100,0 \\
\hline
\end{tabular}

$\mathrm{Na}$ análise da necessidade de profissionais segundo o NAS, obteve-se números decimais que foram arredondados para cima quando o decimal foi maior ou igual a 0,5 e para baixo se menor que 0,4 .

Para averiguar a necessidade de profissionais de enfermagem segundo o NAS foram somadas as pontuações dos pacientes presentes em cada um dos turnos e nas $24 \mathrm{~h}$ em cada dia do estudo. Obtiveram-se (Quadro 2) uma média de 522,5 pontos para o turno da manhã, no turno da tarde, 506,1 pontos e no turno da noite, 561,5 pontos. Em relação à pontuação NAS de 24h obteve-se média de 643,2 pontos.

Quadro 2 - Soma das pontuações NAS, necessidade de profissionais de enfermagem segundopontuação NAS e quadro efetivo de profissionais de enfermagem por turno e nas $24 \mathrm{~h}$ nos períodos de 24 a 30 de junho e 9 a 16 de setembro - São Paulo - 2004

\begin{tabular}{|c|c|c|c|c|c|c|c|c|}
\hline & \multicolumn{2}{|c|}{ Manhã } & \multicolumn{2}{|r|}{ Tarde } & \multicolumn{2}{|r|}{ Noite } & \multicolumn{2}{|r|}{$24 \mathrm{~h}$} \\
\hline & $\begin{array}{l}\text { soma } \\
\text { NAS }\end{array}$ & $\begin{array}{c}\mathrm{n}^{\circ} \mathrm{de} \\
\text { profissionais } \\
\text { segundo NAS }\end{array}$ & $\begin{array}{l}\text { soma } \\
\text { NAS }\end{array}$ & $\begin{array}{c}n^{\circ} \text { de } \\
\text { profissionais } \\
\text { segundo NAS }\end{array}$ & $\begin{array}{l}\text { soma } \\
\text { NAS }\end{array}$ & $\begin{array}{c}n^{\circ} \text { de } \\
\text { profissionais } \\
\text { segundo NAS }\end{array}$ & $\begin{array}{c}\text { Soma } \\
\text { NAS }\end{array}$ & $\begin{array}{c}n^{\circ} \text { de } \\
\text { profissionais } \\
\text { segundo NAS }\end{array}$ \\
\hline dia1 & 513,7 & 5,1 & 554,6 & 5,5 & 542,5 & 5,4 & 617,0 & 6,2 \\
\hline dia2 & 551,5 & 5,5 & 533,0 & 5,3 & 501,6 & 5,0 & 622,0 & 6,2 \\
\hline dia3 & 454,4 & 4,5 & 470,9 & 4,7 & 540,0 & 5,4 & 538,2 & 5,4 \\
\hline dia4 & 472,4 & 4,7 & 488,7 & 4,9 & 600,7 & 6,0 & 641,4 & 6,4 \\
\hline dia5 & 477,4 & 4,8 & 458,9 & 4,6 & 436,4 & 4,4 & 548,6 & 5,5 \\
\hline dia6 & 430,2 & 4,3 & 527,8 & 5,3 & 634,9 & 6,3 & 693,0 & 6,9 \\
\hline dia7 & 493,6 & 4,9 & 468,0 & 4,7 & 440,2 & 4,4 & 540,9 & 5,4 \\
\hline dia8 & 557,7 & 5,6 & 482,3 & 4,8 & 619,3 & 6,2 & 667,4 & 6,7 \\
\hline dia9 & 510,6 & 5,1 & 554,3 & 5,5 & 670,4 & 6,7 & 763,6 & 7,6 \\
\hline dia10 & 579,6 & 5,8 & 517,4 & 5,2 & 587,2 & 5,9 & 695,9 & 7,0 \\
\hline dia11 & 591,0 & 5,9 & 565,9 & 5,7 & 691,6 & 6,9 & 824,0 & 8,2 \\
\hline dia12 & 541,8 & 5,4 & 485,6 & 4,9 & 587,1 & 5,9 & 614,1 & 6,1 \\
\hline dia13 & 546,0 & 5,5 & 535,9 & 5,4 & 587,1 & 5,9 & 672,5 & 6,7 \\
\hline dia14 & 594,7 & 5,9 & 442,5 & 4,4 & 421,6 & 4,2 & 566,0 & 5,7 \\
\hline Média & 522,5 & 5,2 & 506,1 & 5,1 & 561,5 & 5,6 & 643,2 & 6,4 \\
\hline
\end{tabular}


Observa-se que a necessidade NAS por turnos foi de, em média, $5(5,3)$ profissionais de enfermagem e para as $24 \mathrm{~h}$ foi em média de $6(6,4)$ profissionais. Esta diferença se explica pela forma de pontuação, já mencionada, de alguns itens que conferem maior valor a alguns eventos que atingem alta duração ou freqüência nas 24h, mas não nos turnos isoladamente, por exemplo, suporte e cuidado a pacientes e familiares (item 7 do instrumento NAS) ou procedimentos de higiene (item 4), ou, ainda, atividades que ocorreram em apenas um dos turnos, pontuaram no referido turno e também nas 24h como, por exemplo, investigações laboratoriais (item 2).

Tanto nos turnos quanto nas $24 \mathrm{~h}$ a necessidade NAS foi menor do que o quadro efetivo de profissionais presente. Verifica-se, no entanto, uma maior aproximação entre a necessidade NAS e o quadro efetivo médio nas 24 h $(6,4$ e 7,1) do que nos turnos (5,2 e 7,5 para manhã; 5,1 e 7,1 para tarde; 5,6 e 6,9 para noite).

O instrumento, como mencionado, foi construído para aplicação retrospectiva de 24 horas, e mostrou-se, perante os resultados obtidos mais próximo desta aplicação. Verifica-se, porém, que existe a possibilidade de aplicação do NAS por turnos para avaliação de carga de trabalho e classificação de pacientes, desde que se conheça as particularidades que afetam a pontuação, tornando-a menor do que a constatada ao final de $24 \mathrm{~h}$, sendo que, para fins de dimensionamento de pessoal, a pontuação de $24 \mathrm{~h}$ seria mais recomendada.

No entanto, tendo a pesquisadora vivenciado de forma muito próxima a realidade dos referidos turnos e dias durante a coleta dos dados, extremamente trabalhosos, e diante da constatação de uma necessidade de profissionais apontada pelo instrumento NAS como menor do que a realidade, tanto nos turnos quanto nas $24 \mathrm{~h}$, permite tecer algumas considerações:

1. Podem existir processos de trabalho que ocorrem de forma muito própria, com reflexo diferente na carga de trabalho/ consumo de tempo dos profissionais de enfermagem, daquela idealizada na confecção do instrumento, que necessitam ser adequadamente identificadas e melhor enquadradas nos itens de pontuação. Por exemplo, na unidade campo do estudo, a hemodiálise de fluxo lento, que esteve presente por vários plantões, é instalada, controlada e desligada exclusivamente pela enfermeira, que realiza o registro de todos os parâmetros a cada 60/120minutos; dependendo da terapia realiza troca das soluções e bolsas. Ocorre ainda o complemento das soluções utilizadas e, em alguns casos, o preparo total destas soluções, na própria unidade pela enfermeira ou técnico de enfermagem, que corresponde aproximadamente a uma bolsa de 2000 ou 3000ml a cada hora contendo cerca de seis itens, conforme prescrição médica. Este procedimento, quando presente, foi computado como hemodiálise (7,7 pontos), da mesma forma como quando ocorreu hemodiálise convencional ou diálise peritonial con- tínua, que, na realidade da unidade campo de estudo, demandam tempos de assistência diferentes. Talvez o procedimento pudesse, em termos de tempo de assistência, ter sido melhor representado através de uma combinação da pontuação da hemodiálise $(7,7)$ associada a uma pontuação maior no item de registro de monitorização e controles (subitens 1b-12,1 pontos ou 1c-19,6 pontos), dependendo da intensidade.

2. Podem ter ocorrido processos que não foram adequadamente considerados, discriminados, avaliados ou classificados na realização da pontuação dos pacientes durante a coleta dos dados. Por exemplo, existem procedimentos, como curativos, incluídos no item "procedimentos de higiene” que, por serem realizados por enfermeiras e técnicos de enfermagem, podem não ter sido avaliados na totalidade pelos profissionais que foram consultados em separado e talvez não tenham respondido de forma completa quanto ao tempo despendido no procedimento.

3. Poder ter havido aumento na necessidade real da assistência provocado pelo padrão institucional dos processos assistenciais aplicados na realização das atividades, que não pôde ser captada na pontuação, mas que ocasionou a sensação de alta carga de trabalho, mesmo na presença do quadro efetivo típico da unidade. Por exemplo, a realização da mudança de decúbito que no campo de estudo é realizada para a grande maioria dos pacientes a cada 2 horas, obrigatoriamente com a presença de 2 profissionais, no mínimo e para pacientes que se movimentam sozinhos é prestada assistência na mobilização por um profissional o que chega a ocorrer mais de uma vez em uma hora. Assim, sendo o procedimento realizado 3, 12 ou mais vezes ao dia, recebeu mesma pontuação, assim como, se necessitou 3 profissionais 1, 6 ou mais vezes destas mudanças.

4. A produtividade dos profissionais envolvidos no estudo teria sido menor do que a produtividade supostamente considerada no instrumento, de cerca de $80 \%$. Embora não tenha sido essa a percepção, só se poderá confirmar por uma avaliação de produtividade mais detalhada.

\section{CONCLUSÃO}

Na utilização do instrumento, a coleta das informações dos sub-itens 1, 4, 6, 7 e 8 percebeu-se que dificilmente a forma como estas atividades ocorreram teria sido resgatada com fidelidade sem a informação direta do profissional de enfermagem envolvido, o que, teoricamente, compromete a coleta retrospectiva de $24 \mathrm{~h}$, como o instrumento propõe. Ainda, percebeu-se com freqüência a tendência do profissional relatar o cuidado sentido como necessário e não o prestado, sendo estas situações esclarecidas diretamente com os envolvidos durante a coleta. Leva-se a supor que para uso na pontuação de 24 horas, estes itens mereçam uma forma própria de registro onde cada profissional possa 
indicar corretamente, após orientação específica, como e com que duração as atividades ocorreram, já que estas informações não se encontram detalhadas no prontuário. Além disso, durante a pontuação, houve a sensação de que o subitem 7 , suporte e cuidados aos familiares e paciente, deveria conter uma pontuação intermediária entre 4 pontos (7a) dedicação exclusiva por cerca de 1 hora em algum plantão e 32 pontos (7b) - dedicação exclusiva por cerca de 3 horas ou mais em algum plantão. Existe um hiato de pontuação caso a atividade dure entre 1 e 3 horas, tempo considerável na prestação da assistência que, embora tenham ocorrido na realidade deste estudo, foram pontuados como item 7a (4 pontos) por falta de melhor alternativa.

O NAS de 24 h quando aplicado sobre permanências completas, conforme preconizado, resulta em maiores valores, portanto, maior NAS médio. As permanências menores de 24h resultam em NAS mais baixos, porém constituem tempo de assistência considerável da enfermagem.

Podem existir processos de trabalho que ocorrem de forma muito própria, com reflexo no consumo de tempo/ carga de trabalho dos profissionais de enfermagem diferente daquela idealizada na confecção do instrumento e que necessitam ser melhor enquadradas nos itens de pontuação.

\section{REFERÊNCIAS}

1. Tranquitelli AM. Estudo prospectivo para determinação do número de horas de cuidados diretos de enfermagem em uma Unidade de Terapia Intensiva geral [dissertação]. São Paulo: Escola de Enfermagem, Universidade de São Paulo; 1999.

2. Nascimento EFA. Evolução da gravidade de pacientes adultos internados em uma Unidade de Terapia Intensiva [dissertação]. São Paulo: Escola de Enfermagem, Universidade de São Paulo; 2002.

3. Knobel E, Kühl SD. Organização e funcionamento das UTIs. In: Knobel E, editor. Condutas no paciente grave. $2^{\mathrm{a}}$ ed. São Paulo: Atheneu; 1998. p. 1316-31.

4. Correa AK. O paciente em Centro de Terapia Intensiva: reflexão bioética. Rev Esc Enferm USP. 1998;32(4):297-301.

5. Queijo AF. Tradução para o português e validação de um instrumento de medida de carga de trabalho de enfermagem em Unidade de Terapia Intensiva: Nursing Activities Score (NAS). [dissertação]. São Paulo: Escola de Enfermagem, Universidade de São Paulo; 2002.

6. Fein A, Fein SL. Utilization and allocation of critical care resources. In: Shoemaker WC, editor. Textbook of critical care. $4^{\text {th }}$ ed. Philadelphia: Saunders; 2000. p. 2009-15.

7. Matos AJ, editor. Gestão de custos hospitalares: técnicas, análise e tomada de decisão. São Paulo: STS; 2002. A importância da gestão de custos para os hospitais; p. 19-24.
Podem ocorrer processos que não são adequadamente considerados, discriminados, avaliados ou classificados na realização da pontuação dos pacientes.

No entanto, pode-se considerar que o NAS apresentase como interessante e valioso instrumento para classificação de pacientes e avaliação de carga de trabalho para uso da enfermagem em terapia intensiva, uma vez que o quantitativo de profissionais de enfermagem sugerido, pela pontuação NAS, está muito próximo ao da realidade encontrada no campo de estudo, considerado, internacionalmente, como sendo local de boas práticas de enfermagem e que além disso foi objeto de investigação para elaboração do instrumento Nursing Activities Score (NAS) desenvolvido por Miranda e seus colaboradores ${ }^{(10)}$.

Assim, diante destas considerações, surgem novas perspectivas para estudos a serem desenvolvidos no sentido de clarificar a aplicabilidade deste instrumento de quantificação de carga de trabalho e dimensionamento de pessoal em UTI, a fim de que possa ser utilizado de forma consciente, para classificação de pacientes segundo carga de trabalho e dimensionamento de pessoal, orientado para busca da qualidade assistencial de enfermagem.

8. Gaidzinski RR. Dimensionamento de pessoal de enfermagem em instituições hospitalares [tese livre-docência] São Paulo: Escola de Enfermagem, Universidade de São Paulo; 1998.

9. Gaidzinski RR. Dimensionamento do pessoal de enfermagem. In: Kurcgant P, coordenadora. Administração em enfermagem. São Paulo: EPU; 1991. p. 91-6.

10. Miranda DR, Nap R, Rijk A, Schaufeli W, Iapichino G. Nursing activities score. Crit Care Med. 2003;31(2):374-82.

11. Miranda DR, Rijk A, Schaufeli W. Simplified therapeutic intervention scoring system: the TISS-28 itens results from a multicenter study. Crit Care Med. 1996;24(1):64-73.

12. Le Gall JR, Lemeshow S, Saulnier F. A new Simplified Acute Physiology Score (SAPS II) based on a European/North American multicenter study. JAMA. 1993;270(24):2957-63.

13. Société Française d'Anesthésie et de Réanimation. Scoring systems for ICU and surgical patients. SAPSII and predicted mortality [text on the Internet]. [cited 2005 Apr 27]. Available from: <http//www.sfar.org/scores2/saps2.html\#def> 